\title{
Preventive Care in Nursing and Midwifery Journal
}

2018; 8(2): $1-8$

\author{
Factors Associated With medication Errors in the Psychiatric Ward of \\ Razi Hospital in Tabriz: Perspectives of Nurses \\ Abdi $\mathrm{M}^{1} \oplus$, Piri $\mathrm{Sh}^{2} \oplus$, Mohammadian $\mathrm{R}^{2} \varpi$, Asadi Aghajeri $\mathrm{M}^{2} \varpi$, khademi $^{3 *}$ \\ ${ }^{1}$ Department of Emergency and Critical Care, School of Nursing and Midwifery, Zanjan University of Medical Sciences, Zanjan, Iran \\ ${ }^{2}$ MSc. Department of Nursing, Maragheh Branch, Islamic Azad University, Maragheh, Iran \\ ${ }^{* 3}$ Dept. Nursing, Maragheh Branch, Islamic Azad University, Maragheh, Iran
}

*Corresponding Author: Dept. Nursing, Maragheh Branch, Islamic Azad University, Maragheh, Iran

Email: Elmira.khademi@yahoo.com

Received: 7 Jan $2019 \quad$ Accepted: 11 Aug 2019

\begin{abstract}
Background: Medication errors are considered to be the most significant safety threatening factors for the patients in hospital, to which many factors contribute.

Objectives: This study was conducted to determine the role of associated factors in the incidence of medication errors in the psychiatric ward of Razi Hospital in Tabriz from the perspectives of nurses in 2017.

Methods: In this descriptive cross-sectional study, we selected 150 nurses working in the psychiatric ward of Razi Hospital in Tabriz through a random sampling method. The data collection instrument included a demographic and researcher-made questionnaire to assess the effective factors in the incidence of medication errors according to the perspectives of nurses. The data were imported into SPSS and analyzed via ANOVA and Chi-square tests.

Results: In this study, 95 (63.3\%) women and 55 (36.7\%) men with the mean age of $34.4 \pm 0.66$ participated. The highest mean score for the causes of medication errors was related to the professional errors made by nurses $(33.93 \pm 2.61)$ and the structure of the psychiatric ward $(27.96 \pm 5.8)$. The change in Kardex during the transfer of the patient to other wards was the most significant cause of errors with a mean of $4.35 \pm 5.53$. The mean score of medication errors was significantly different from level of education, age, work experience, and employment types. However, it was not significantly different from gender, marital status, nurse job position, and shift work rotation.

Conclusion: Considering the incidence of the most common medication errors in the field of nursing careers and structure of psychiatric wards, we recommend that nurse managers increase the skills of medication administration through holding training courses and improve the physical conditions of the ward.
\end{abstract}

\section{Keywords: medication errors, nurses, psychiatric ward}

\section{Introduction}

Providing patient safety is one of the most important tasks of healthcare -medical complexes. Today, patient safety in the delivery service system is a key concept and is regarded as one of the important indicators of control quality of health services [1]. Factors such as wrong injection, falls, burns, and errors in invasive procedures threaten patients' safety, among which medication errors are the most important factors [2]. Medication errors are defined as deviations from the proper conduct of the treatment process, which may occur in administration, preparation, delivery, use, or distribution of drugs. Administering drugs to patients is one of the main tasks of nurses and the most important part of care 
processes. Proper administration of drugs to patients requires high level of knowledge and accuracy on the part of nurses [3]. Today, various types of medicine are used in the field of health and treatment of patients, all of which may have harmful effects on the health of individuals despite their beneficial effects. Thus, nurses should be aware of the importance of correctly recognizing and administering drugs to prevent possible complications due to medication errors [4].

According to a survey conducted in England in 2018, more than 2 million people are injured each year due to medication errors, and of which about 100,000 die in hospitals [5]. According to previous studies, the cost of medication side effects was estimated to be nearly $\$ 300$ million in 2018 [6]. However, it is difficult to estimate accurate statistics in the third world and developing countries due to the lack of proper registration and reports [7]. Watanabe et al. showed that $30 \%$ of the injured patients due to medication errors are impaired for more than 6 months or even lose their lives [8]. In this regard, psychiatric drugs are very sensitive, and if administered incorrectly, they poison the patient very early. A majority of medicines for patients with psychological problems are taken orally, and due to drug similarities, the risk of wrong administration is high [10]. The structure of the psychiatric ward, the lack of co-operation of mental patients in treatments, the poisoning dose close with the therapeutic dose in moodstabilizing, antipsychotic, and depression drugs necessitate nurses' attention when administering the drug $[11,12]$. Ferrera et al. mentioned that more than half of the medication errors could be prevented, and observing the precise principles of drugs medication could reduce the incidence of errors [13].

The reason for the incidence of medication errors is not limited to one aspect and must be controlled in all aspects [14]. There are several factors involved in the incidence of medication errors; however, in previous studies, the cause of medication errors has been differently reported. For instance, Jones et al. have reported that the most common cause of medication errors made by nurses is the lack of compliance with the five rights of medication administration (the right patient, the right drug, the right dose, the right route, and the right time) [15]. However, Sarvadikar et al. (2010) stated that most of the errors occurred in the process of administering drugs to the patients [16]. A study by Cottney et al in 2014 suggested that medication errors of the psychiatric ward is more likely to occur due to insufficient knowledge and skills of nurses in terms of the right dose, time, and medication [12]. Yakubi et al. and Mashhala et al. reported factors such as the burden on nurses, the lack of readability of drug orders in patient's file or medication card, doctors' bad handwriting, the similarity in the form and packages of drugs, fatigue due to overwork, anxiety and stress resulted from work, noise of the ward settings, and nurses' dissatisfaction with the amount of salary and benefits as the most important causes of medication errors [17,18]. In summary, the main causes of the incidence of nurses' medication errors are workplaces, drug companies, and nurse management. Each study has presented a cause for the error in a different way, and the number of studies that comprehensively examine these factors is small. It seems that there has not been a study in Iran to examine the medication errors of the psychiatric ward.

In the present study, considering the complications of drugs for patients, heavy economic costs for individuals and society due to medication errors, the role of each member in the drug supply chain in the incidence of medication errors, and the sensitivity of drugs in the psychiatric ward, we aim to determine the associated factors in the incidence of medication errors in the psychiatric ward of Razi Hospital in Tabriz from the perspective of nurses. We hope that the results of this study can help identify the causes of the incidence of medication errors and provide a solution to reduce their incidence and complications in patients and decrease the cost of treatment and the duration of hospitalization.

\section{Methods}

This descriptive cross-sectional study was carried out using random sampling method based on a random table of numbers in the first half of 2017. One hundred fifty nurses working in the psychiatric ward of Razi psychiatric hospital participated in the present study. The main population of the study included 280 nurses. To 
estimate the minimum sample size at $95 \%$ confidence level, with a $90 \%$ test power and the mean $n=\frac{z_{1}-\frac{\alpha}{x}}{d^{x}}$ incidence of medication errors as at least $p=0.18$

using the formula $(\mathrm{d}=1 / 96)$, the final sample size included 141 participants. To prevent sample loss, 165 people were invited to participate in the study. During the research, 15 people refused to continue, and the study was done using 150 people.

The data was collected in morning, noon, and night shifts. The inclusion criteria in this study were having more than one year of work experience in hospital, undergraduate and postgraduate studies, and lack of mental and physical illness. Nurses who had less than one year of work experience or were reluctant to participate in the study were excluded. In order to ensure the confidentiality of information, it was announced to the participants that writing name and surname is optional.

This study was approved by the Ethics Committee of Maragheh University of Medical Sciences with number 5/13/15/12378 and Iranian code of IR.MARAGHEHPHC.REC.1396.26. After obtaining informed consent from the patients, compliance with the ethical issues according to the Helsinki Treaty, and compliance with the conditions of inclusion criteria, the questionnaires were completed.

A demographic and researcher-made questionnaire was used to assess the factors affecting the incidence of medication errors according to the perspective of nurses, which were designed after studying different texts $[19,20]$. The questionnaire consists of 31 questions and five areas in which questions 1 to 10 are related to nursing professional errors, 11 to 18 to the conditions of the ward and the attendance of patients, 19 to 22 to doctors' errors, 23 to 24 to the errors of drug companies, and 25 to 31 to the management process errors. The scoring procedure of the questionnaire was considered as a 5-point Likert spectrum that included very low, low, moderate, high, and very high with the scores of 1 to 5 . The least acquired score is 31 , and the maximum is 155. Higher scores in one area indicate the importance of that area. The face and content validity of this questionnaire was verified by ten faculty members and nursing experts. To determine the reliability, split-half method and the correlation coefficient calculation were used in two parts of the questionnaire, for which correlation coefficient was found to be $81 \%$.

The data were imported into SPSS version 21 software, and then the data normalization was determined using Kolmogorov-Smirnov test. To analyze the data, descriptive statistical indices were employed. Besides, to compare the sociodemographic characteristics with the probability of medication errors, statistical analysis of variance and Chi-square were used.

\section{Results}

In this study, 95 (63.3\%) women and $55(36.7 \%)$ male participated. The mean age of the participants was $34.4 \pm 0.69$ years. The mean total score of medication errors was $106.25 \pm 9.28$. The highest mean score for nurses' professional errors was $33.93 \pm 2.61$, and factors associated with the ward was $27.96 \pm 5.8$ (Table 1$)$. 
Factors associated with medication errors in the ....

Table 1: The frequency of effective factors in the incidence of medication errors from the perspective of nurses in the psychiatric ward of Razi Hospital in Tabriz

\begin{tabular}{|c|c|c|c|c|}
\hline \multicolumn{2}{|c|}{ Demographic Factors } & \multirow{2}{*}{$\begin{array}{c}\mathbf{N}(\%) \\
55(36 / 7) \\
\end{array}$} & \multirow{2}{*}{$\begin{array}{c}\mathbf{M} \pm \text { SD } \\
106 / 81 \pm 7 / 97 \\
\end{array}$} & \multirow{3}{*}{$\begin{array}{l}\text { P Value } \\
{ }^{*} \mathrm{p}=0 / 586\end{array}$} \\
\hline & Female & & & \\
\hline Gender & Male & $95(63 / 3)$ & $105 / 69 \pm 10 / 01$ & \\
\hline \multirow{2}{*}{ Education Level } & Bachelor of Science & $125(83 / 3)$ & $109 / 75 \pm 9 / 53$ & \multirow{2}{*}{${ }^{*} \mathrm{p}<0 / 001$} \\
\hline & Master of Science & $25(16 / 7)$ & $88 / 92 \pm 7 / 52$ & \\
\hline \multirow{2}{*}{ Marital Status } & Single & $70(46 / 7)$ & $106 / 94 \pm 8 / 68$ & \multirow{2}{*}{${ }^{*} \mathrm{p}=0 / 209$} \\
\hline & Married & $80(53 / 3)$ & $105 / 60 \pm 9 / 81$ & \\
\hline \multirow{2}{*}{ Job Position } & Nurse & $145(96 / 7)$ & $106 / 27 \pm 9 / 45$ & \multirow{2}{*}{${ }^{*} \mathrm{p}=0 / 837$} \\
\hline & Head Nurse & $5(3 / 3)$ & $105 / 01 \pm 9 / 45$ & \\
\hline \multirow{2}{*}{ Work Shift } & Fixed Shift & $45(30)$ & $105 / 60 \pm 5 / 03$ & \multirow{2}{*}{${ }^{*} \mathrm{p}=0 / 284$} \\
\hline & Rotational & $105(70)$ & $106 / 38 \pm 10 / 58$ & \\
\hline \multirow{3}{*}{ Age } & $25-35$ & $95(63 / 3)$ & $106 / 12 \pm 10 / 34$ & \multirow{3}{*}{${ }^{* *} \mathrm{p}=0 / 04$} \\
\hline & $35-45$ & $45(30)$ & $101 \pm 7 / 18$ & \\
\hline & $45-50$ & $10(6 / 7)$ & $98 \pm 3 / 16$ & \\
\hline \multirow{3}{*}{ Work Experience } & $10>$ & $115(76 / 7)$ & $106 / 40 \pm 9 / 84$ & \multirow{3}{*}{${ }^{* *} \mathrm{p}=0 / 01$} \\
\hline & $10-20$ & $25(16 / 7)$ & $98 / 60 \pm 6 / 31$ & \\
\hline & $20<$ & $10(6 / 7)$ & $99 \pm 3 / 16$ & \\
\hline \multirow{5}{*}{$\begin{array}{c}\text { Recruitment } \\
\text { Status }\end{array}$} & Draft & $20(13 / 3)$ & $107 / 75 \pm 18 / 85$ & \multirow{5}{*}{${ }^{* *} \mathrm{p}=0 / 021$} \\
\hline & Hiring & $15(10)$ & $106 / 33 \pm 6 / 77$ & \\
\hline & Contractual & $75(50)$ & $104 / 76 \pm 7 / 27$ & \\
\hline & Official Trial & $20(13 / 3)$ & $96 / 42 \pm 6 / 4$ & \\
\hline & Official & $20(13 / 3)$ & $92 / 50 \pm 2 / 66$ & \\
\hline *Chi-square & ** one way ANOVA & & & \\
\hline
\end{tabular}

The most important cause of nursing professional errors was related to non-compliance with the eight rights of medication errors with a mean of 3.70 \pm 0.97 . In the factors related to the ward, the most important reason was the change of Kardex when the patient was transferred to other wards with a mean of $4.53 \pm 5.58$. Regarding the factors related to nursing management, the most leading cause was inappropriate work shift layout of nurses with the mean of $3 / 53+1 / 02$. The association between the mean score of medication errors and other socio-demographics of participants is shown in Table 2. There was a significant difference between the mean score of medication errors and that of the level of education, age, increase in work experience, and type of employment $(\mathrm{p}<0.05)$. However, there was no significant difference between medication errors and sex, marital status, and nurses' job position and work shift rotation $(\mathrm{p}<0.05)$. 
Abdi M, Piri Sh, Mohammadian R, Asadi Aghajeri M, khademi E..... 5

Table 2: Comparison of the mean score of medication errors with individual-social factors

\begin{tabular}{|c|c|c|c|c|c|c|c|c|}
\hline \multirow{2}{*}{ 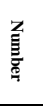 } & \multirow{2}{*}{ Field } & \multirow{2}{*}{ Questions } & $\begin{array}{l}\text { Very } \\
\text { Low }\end{array}$ & Low & Moderate & High & Very High & \multirow{2}{*}{$\mathrm{M} \pm \mathrm{SD}$} \\
\hline & & & $\mathbf{N}(\%)$ & $\mathbf{N}(\%)$ & $\mathbf{N}(\%)$ & $\mathbf{N}(\%)$ & $\mathbf{N}(\%)$ & \\
\hline 1 & \multirow{11}{*}{ 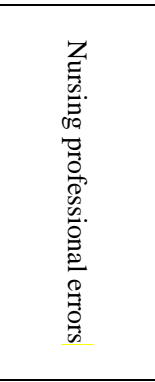 } & Lack of sufficient knowledge about medicine & $(0) 0$ & $(33 / 3) 50$ & (30) 45 & $(23 / 3) 35$ & $(13 / 3) 20$ & $3 / 16 \pm 1 / 03$ \\
\hline 2 & & Indifference of nurses to their profession & $(0) 0$ & $(16 / 7) 25$ & (40) 60 & $(33 / 3) 50$ & (10) 15 & $3 / 36 \pm 0 / 87$ \\
\hline 3 & & Low salary and economic problem & $(0) 0$ & $(16 / 7) 25$ & $(36 / 7) 55$ & $(46 / 7) 70$ & $(0) 0$ & $3 / 30 \pm 0 / 73$ \\
\hline 4 & & Nurses' family challenges & $(0) 0$ & $(20) 30$ & $(30) 45$ & $(33 / 3) 50$ & $(16 / 7) 25$ & $3 / 46 \pm 0 / 99$ \\
\hline 5 & & Nurses' psychological challenges & $(0) 0$ & $(313) 20$ & $(43 / 3) 65$ & $(23 / 3) 35$ & $(20) 30$ & $3 / 50 \pm 0 / 96$ \\
\hline 6 & & Much workload & $(0) 0$ & $(10) 15$ & $(30) 45$ & $(50) 75$ & $(10) 15$ & $3 / 60 \pm 0 / 80$ \\
\hline 7 & & Failure to comply with 8 rights of medication administration & $(0) 0$ & $(16 / 7) 25$ & $(16 / 7) 25$ & $(46 / 7) 70$ & $(20) 30$ & $3 / 70 \pm 0 / 97$ \\
\hline 8 & & Error in the rate of drug infusion & $(3 / 3) 5$ & $(23 / 3) 35$ & $(33 / 3) 50$ & $(33 / 3) 50$ & $(6 / 7) 10$ & $3 / 16 \pm 0 / 97$ \\
\hline 9 & & Forgetting medication administration in due time & $(6 / 7) 10$ & (10) 15 & $(36 / 7) 55$ & $(40) 60$ & $(6 / 7) 10$ & $3 / 30 \pm 0 / 97$ \\
\hline \multirow{2}{*}{10} & & \multirow{2}{*}{ Failure to properly transfer physician's orders to Kardex } & $(0) 0$ & $(20) 30$ & $(30) 45$ & $(43 / 3) 65$ & $(6 / 7) 10$ & $3 / 36 \pm 0 / 87$ \\
\hline & & & \multicolumn{6}{|c|}{$33 / 93 \pm 2 / 61$} \\
\hline 11 & \multirow{9}{*}{ 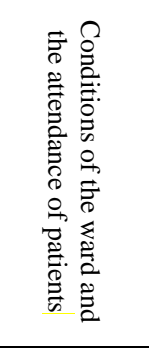 } & Lack of appropriate drug information (DI) resources in the wards & $(0) 0$ & $(26 / 7) 40$ & (30) 45 & $(33 / 3) 50$ & (10) 15 & $3 / 26 \pm 0 / 96$ \\
\hline 12 & & $\begin{array}{c}\text { The physical conditions of the ward in terms of light, ventilation, } \\
\text { temperature, } \ldots\end{array}$ & $(0) 0$ & $(13 / 3) 20$ & $(46 / 7) 70$ & $(33 / 3) 50$ & $(6 / 7) 10$ & $3 / 33 \pm 0 / 79$ \\
\hline 13 & & Noise and crowded setting of the ward & $(0) 0$ & $(13 / 3) 20$ & (40) 60 & $(36 / 7) 55$ & (10) 15 & $3 / 43 \pm 0 / 84$ \\
\hline 14 & & Placement of the drugs on the shelves & $(0) 0$ & $(16 / 7) 25$ & $(33 / 3) 50$ & $(33 / 3) 50$ & $(16 / 7) 25$ & $3 / 50 \pm 0 / 96$ \\
\hline 15 & & The large variety of drugs in the ward & $(0) 0$ & $(6 / 7) 10$ & $(53 / 3) 80$ & (30) 45 & (10) 15 & $3 / 43 \pm 0 / 76$ \\
\hline 16 & & Attendance of patient's companions in the ward & $(0) 0$ & $(26 / 7) 40$ & (40) 60 & $(23 / 3) 35$ & (10) 15 & $3 / 16 \pm 0 / 93$ \\
\hline 17 & & Patients and companions, inappropriate behavior & $(0) 0$ & $(13 / 3) 20$ & $(46 / 7) 70$ & $(36 / 7) 55$ & $(3 / 3) 5$ & $3 / 30 \pm 0 / 73$ \\
\hline & & & $(0) 0$ & $(23 / 3) 35$ & $(20) 30$ & $(40) 60$ & $(16 / 7) 25$ & $4 / 53 \pm 5 / 58$ \\
\hline 18 & & Change of Kardex when transferring the patient to other wards & \multicolumn{6}{|c|}{$27 / 96 \pm 5 / 8$} \\
\hline 19 & \multirow{5}{*}{ 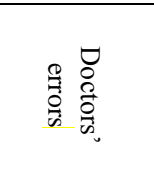 } & Giving instructions on the phone by doctors & $(0) 0$ & (10) 15 & $(43 / 3) 65$ & $(40) 60$ & $(6 / 7) 10$ & $3 / 43 \pm 0 / 76$ \\
\hline 20 & & Illegibility of doctor's handwriting & $(0) 0$ & $(16 / 7) 25$ & $(43 / 3) 65$ & $(30) 45$ & (10) 15 & $3 / 33 \pm 0 / 87$ \\
\hline 21 & & Error in prescribing drugs & $(0) 0$ & $(23 / 3) 35$ & $(23 / 3) 35$ & $(40) 60$ & $(13 / 3) 20$ & $3 / 43 \pm 0 / 99$ \\
\hline \multirow[t]{2}{*}{22} & & Failure to comply with the appropriate time for prescribing the & $(0) 0$ & $(24 / 1) 35$ & (31) 45 & (31) 45 & $(13 / 8) 20$ & $3 / 34 \pm 0 / 99$ \\
\hline & & drugs by doctor & \multicolumn{6}{|c|}{$13 / 54 \pm 1 / 7$} \\
\hline 23 & \multirow{11}{*}{ 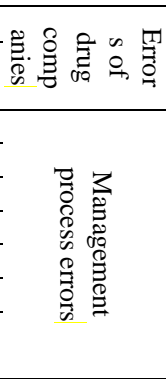 } & Inappropriate forms and naming of drugs & $(0) 0$ & $(16 / 7) 25$ & $(36 / 7) 55$ & $(33 / 3) 50$ & $(13 / 3) 20$ & $3 / 43 \pm 0 / 92$ \\
\hline \multirow{2}{*}{24} & & \multirow{2}{*}{ Pharmaceutical similarities in terms of form, name and ... } & $(3 / 3) 5$ & $(13 / 3) 20$ & $(33 / 3) 50$ & $(36 / 7) 55$ & $(13 / 3) 20$ & $3 / 43 \pm 0 / 99$ \\
\hline & & & \multicolumn{6}{|c|}{$6 / 86 \pm 1 / 15$} \\
\hline 25 & & Inappropriate relationship of nurses with ward authorities & $(0) 0$ & $(20) 30$ & $(33 / 3) 50$ & $(43 / 3) 65$ & $(3 / 3) 5$ & $3 / 30 \pm 0 / 82$ \\
\hline 26 & & The existence of occupational discrimination & $(0) 0$ & $(6 / 7) 10$ & $(46 / 7) 70$ & $(43 / 3) 65$ & $(3 / 3) 5$ & $3 / 43 \pm 0 / 66$ \\
\hline 27 & & Lack of recording and error reporting systems & $(0) 0$ & $(16 / 7) 25$ & $(43 / 3) 65$ & $(33 / 3) 50$ & $(6 / 7) 10$ & $3 / 30 \pm 0 / 82$ \\
\hline 28 & & The inappropriate layout of nurses' work shift & $(0) 0$ & $(16 / 7) 25$ & $(36 / 7) 55$ & $(23 / 3) 35$ & $(23 / 3) 35$ & $3 / 53 \pm 1 / 02$ \\
\hline 29 & & Shortage of nurses in proportion to the number of patients & $(0) 0$ & $(13 / 3) 20$ & $(43 / 3) 65$ & $(30) 45$ & $(13 / 3) 20$ & $3 / 43 \pm 0 / 88$ \\
\hline 30 & & Lack of supervising care processes by ward authorities & $(3 / 3) 5$ & $(6 / 7) 10$ & $(36 / 7) 55$ & $(43 / 3) 65$ & (10) 15 & $3 / 50 \pm 0 / 88$ \\
\hline \multirow{3}{*}{31} & & & $(0) 0$ & $(13 / 3) 20$ & $(36 / 7) 55$ & $(40) 60$ & (10) 15 & $3 / 46 \pm 0 / 84$ \\
\hline & & The presence of a large number of serious patients in the ward & \multicolumn{6}{|c|}{$23 / 96 \pm 2 / 64$} \\
\hline & & Mean total score of medication errors & & & & $25 \pm 9 / 28$ & & \\
\hline
\end{tabular}

\section{Discussion}

In this study, the role of effective factors in the incidence of nurses' medication errors was examined. We found that the most medication errors were related to Kardex change when the patient was transferred to other wards as well as non-compliance with the 8 rights of medication administration which is related to nursing field and disorder of the ward. Katney et al., in a prospective observational study entitled "survey of medical errors in urban psychiatric hospital", reported that the most common medication errors were amnesia in giving correct amount of drug (37\%), incorrect method (18\%), incorrect form (12\%) and incorrect times (9\%) in British hospitals [12]. In fact, this study points to nurses' failure to properly check drug and noncompliance with the 8 rights of medication administration, which are consistent with the results of the present study. The strength of this study was its observational survey of 4177 Pharmaceutical cares by nurses and the report of 139 errors. Although the observational study can be very objective to examine the care, it may unconsciously remind the caregiver of the observer, increase the quality of his/her work, and cause a bias in the research. Therefore, elimination of these confounding factors must be carefully done, while Katney et al. did not point out how to remove this bias.

Sorensen et al. (2013), in a cross-sectional observational study entitled" medication errors in 
the psychiatric ward" and after observing 1082 cases drug administration, reported 189 errors. The most common medication errors were nurses' professional skills in administrating medication (75\%), wrong prescription (10\%), error in prescribing discharge $(10 \%)$, and illegibility and incomprehensibility of physicians' prescription $(5 \%)$. The most common medication errors in this study are related to nurses' skills, which is consistent with the present study. This study has not paid attention to the various aspects of nursing care during medication such as conditions of the ward, mental status of nurses, and other individual and social factors. The volume of care review is lower than that of other observational studies. Due to the use of different instruments in checking medication errors, the mean score of medication errors was not comparable in various studies.

Julaei et al. (2016), in a cross-sectional study, using a population of 300 nurses working in hospitals affiliated with Tehran University of Medical Sciences, reported that the most common types of medication errors were drug administration later or earlier than the due time, failure to take necessary measures before administering drugs, which agrees with findings of the present study [22]. The strength of this study was using a high volume of samples and studying the conditions of work environment via an independent questionnaire. The difference between this study and the presentt study was in the use of the type of medication errors-checking instruments. Julia et al. used a researcher-made questionnaire to examine the medication errors related to nurses' professional performance, and other areas of errors were not addressed. For example, in the present study, one of the biggest medication errors happened due to the physical conditions of the ward, while but Julaei et al. reported that the conditions of the nurses' work environment were favorable for pharmaceutical care [22]. Besides, Julaei et al. did not address the physical conditions of the ward, the placement of medicines in the pharmacist, the presence of companions in the ward, and noises, which could be the reason for the differences between the two studies.

Jones et al., in a cross-sectional study, reported that the most common cause of the incidence of medication errors from the perspective of the nurses was non-compliance with five rights of medication administration (the right patient, the right medication, the right dose, the right method, and the right time), which is consistent with the present study [24]. The similarity between the study of Jones et al. and the present study was in the sample size and error analysis from the perspective of nurses. This study has used five rights of medication administration to examine errors, while, in the present study, eight rights of medication administration (the right patient, the right medication, the right dose, the right route, the right time, the right administration, the right recording and the right patient response to medication) were used, which is a newer form of this law. Mi-Ae You et al., (2015) in a crosssectional study of 312 nurses in three South Korean hospitals, noted that most of the incidences of medication errors were due to the shortage of nurses in a work shift. Mi-Ae You used a 29-item questionnaire focused more on drug similarities, and little attention was paid to nursing management, medical errors, and conditions of the ward. Moreover, there was no proper distinction among the causes of medication errors. However, the present study analyzed the role of other factors in nursing errors via dividing the nurses' medication errors into five areas.

In the present study, there was a significant difference between the mean score of medication errors and the increase in education level, age, work experience, and type of employment, while no significant difference was found between the mean score of medication errors and that of sex, marital status, nursing job position, and work shift rotation. Shouhani et al, using a sample of 120 nurses, found that the mean score of medication errors was not associated with an increase in educational level, work-related experience, gender, marital status, and age, all of are consistent with the present study except for age factor [23]. However, Yaghoubi et al, using 127 nurses of Zahedan hospital, showed that the probability of making errors by nurses with work shift rotation is more than the one in others, which is in contrast with the present study [17]. In their study, the minimum working experience was three months, and this could be the reason for the difference in the results the two studies.

Nursing Professional errors and physical conditions of the ward play a major role in nursing staff's medication errors. Considering the 
incidence of medication errors in cases such as Kardex change when transferring patients to other wards, failure to comply with eight rights of medication administration, overwork, psychological challenges of nurses, job discriminations, and lack of supervision on care processes by ward authorities, we suggest that job training, in the form of continuous educational courses, should be offered to nurses to increase their knowledge and performance and to nurse managers for more effective planning. Due to the existence of errors such as the placement of drugs on the shelves and the noise and crowded environment of the ward, hospital administrators are recommended to improve the physical conditions of the workplace. Based on the possibility of an error due to similarities of form and verbal of drugs, it would be more effective to design a system to check the similarity of drugs before issuing a license in food and drug administration. Finally, hospital administrators need to oversee doctors' handwriting and the way of writing a prescription.

One of the limitations of this study was its use of only one hospital for sampling due to the lack of other psychiatric hospitals in Tabriz. Another limitation was the lack of comparisons between psychiatric wards and special, surgery, and emergency wards due to the specificity of the psychiatric hospital. Some of the medication errors were due to the delayed response of medications due to the carelessness of pharmaceutical companies in manufacturing medicines, and there were not enough facilities and opportunities to study these issues in the research. Another limitation was the use of selfreports as the data collection tool.

\section{Acknowledgments}

The authors appreciate the staff of the psychiatric wards of Razi Hospital in Tabriz, who participated in this study despite their heavy work loads. We also express our special thanks to the authorities of Tabriz University of Medical Sciences, Maragheh Medical Sciences, and Maragheh Islamic Azad University, who provided the basis of this study.

\section{Conflict of interest}

The authors of this article declare that there is no conflict of interest in writing this article.

\section{References}

1. Tanti A, Camilleri M, Borg AA, et al. Opinions of Maltese doctors and pharmacists on medication errors. Int J Risk Saf Med. 2017; 29(1-2): 81-99.

2. Sohrevardi SM, Jarahzadeh MH, Mirzaei E, et al. Medication errors in patients with enteral feeding tubes in the intensive care unit. J Res pharm pract. 2017; 6(2): 100-105.

3. Morales-Gonzalez MF, Galiano Galvez MA. Predesigned labels to prevent medication errors in hospitalized patients: a quasi-experimental design study. Medwave. 2017;17(8): 7038.

4. Mansouri A, Ahmadvand A, Hadjibabaie M, et al. A review of medication errors in iran: sources, underreporting reasons and preventive measures. Iran J Pharm Res. 2014; 13(1):3.

5. Elliott R, Camacho E, Campbell F, et al. Prevalence and economic burden of medication errors in the NHS in England. Rapid evidence synthesis and economic analysis of the prevalence and burden of medication error in the UK. 2018.

6. Feleke SA, Mulatu MA, Yesmaw YS. Medication administration error: magnitude and associated factors among nurses in Ethiopia. BMC Nurs. 2015; 14(1): 53.

7. Cheragi MA, Manoocheri H, Mohammadnejad E, Ehsani SR. Types and causes of medication errors from nurse's viewpoint. Iran $\mathbf{J}$ Nurs Midwifery Res. 2013; 18(3): 228-31.

8. Watanabe JH, McInnis T, Hirsch JD. Cost of prescription drug-related morbidity and mortality. Ann Pharmacother. 2018; 52(9): 829-37.

9. Cipriani A, Furukawa TA, Salanti G, et al. Comparative efficacy and acceptability of 21 antidepressant drugs for the acute treatment of adults with major depressive disorder: a systematic review and network meta-analysis. Lancet. 2018; 391.

10. Haw C, Stubbs J, Dickens GL. Barriers to the reporting of medication administration errors and near misses: an interview study of nurses at a psychiatric hospital. J Psychiatr Ment Health Nurs. 2014; 21(9): 797-805.

11. Oruch R, Elderbi MA, Khattab HA, Pryme IF, Lund A. Lithium: a review of pharmacology, clinical uses, and toxicity. Eur J Pharmacol. 2014; 740: 464-73.

12. Cottney A, Innes J. Medication-administration errors in an urban mental health hospital: A direct observation study. Int $\mathbf{J}$ Mental Health Nurs. 2015; 24(1): 65-74. 
13. Ferrah N, Lovell JJ, Ibrahim JE. Systematic review of the prevalence of medication errors resulting in hospitalization and death of nursing home residents. J Am Geriatr Soc. 2017; 65(2): 433-42.

14. Ruiz ME, Suñol MM, Miguélez JR, et al. Medication errors in a neonatal unit: One of the main adverse events. An Pediatr (Barc). 2016; 84(4): 211-17.

15. Jones JH, Treiber L. When the 5 rights go wrong: medication errors from the nursing perspective. J Nurs Care Qual. 2010; 25(3): 24047.

16. Sarvadikar A, Prescott G, Williams D. Attitudes to reporting medication error among differing healthcare professionals. Eur J Clin Pharmacol. 2010; 66(8): 843-53.

17. Yaghoobi M NA, CHarkhat Gorgich EAH, Salehinya H. Nurses' Perspectives Of The Types And Causes Of Medication Errors. Iran J Nurs. 2015; 28(93,94): 1-10.

18. Mosahneh A, Ahmadi B, Akbarisari A, Rahimi Foroshani A. Assessing the Causes of Medication Errors from the Nurses' Viewpoints of Hospitals at Abadan City in 2013. J Hospital. 2016; 15(3): 41-51.

19. Tang FI, Sheu SJ, Yu S, Wei IL, Chen CH. Nurses relate the contributing factors involved in medication errors. J Clin Nurs. 2007; 16(3): 44757.

20. Hosseinzadeh M, Ezate Aghajari P, Mahdavi N. Reasons of Nurses' Medication Errors and Persepectives of Nurses on Barriers of Error Reporting. Hayat. 2012; 18(2): 66-75.

21. Soerensen AL, Lisby M, Nielsen LP, Poulsen BK, Mainz J. The medication process in a psychiatric hospital: are errors a potential threat to patient safety? Risk Manag Healthc Policy. 2013; 6: 23-31.

22. Joolaee S, Shali M, Hooshmand A, Rahimi S, Haghani $\mathrm{H}$. The relationship between medication errors and nurses' work environment. Med Surg Nurs J. 2016; 4(4): 27-34.

23. Shohani $M$, Tavan $H$. Factors affecting medication errors from the perspective of nursing staff. J Clin Diagn Res. 2018; 12 (3): IC01-IC04.

24. Jones JH, Treiber L. When the 5 rights go wrong: medication errors from the nursing perspective. J Nurs Care Qual. 2010; 25(3): 24047.

25. You MA, Choe MH, Park GO, Kim SH, Son YJ. Perceptions regarding medication administration errors among hospital staff nurses of South Korea. Int J Qual Health Care. 2015; 27(4): 276-83. 